\title{
Liquid Course Artifacts Software Platform
}

\author{
Marcos Baez ${ }^{1}$, Boualem Benaltallah ${ }^{2}$, Fabio Casati ${ }^{1}$, Van M. Chhieng ${ }^{2}$, \\ Alejandro Mussi ${ }^{1}$, Qamal Kosim Satyaputra ${ }^{2}$ \\ 1 Dipartimento di Ingegneria e Scienza dell'Informazione, University of Trento, Italy \\ ${ }^{2}$ School of Computer Science and Engineering, UNSW, Sydney, Australia
}

\begin{abstract}
Abstract Liquid Course Artifacts Software Platform aims to improve social productivity and enhance interactive experience for teaching and collaborating by using suppliment materials such as slides, exercises, audios, videos and books.
\end{abstract}

\section{Introduction}

Teaching a course involves preparing notes, tutorials, exercises, exams, slides and others teaching materials. It is very common that besides preparing the core teaching artifacts such as notes and tutorials, it is useful to provide access to complementary materials that add to the student experience and improve learning such as additional non conventional supplement materials (exercises, slides, books, tags, audios, videos).

Services like Wikipedia, Q\&A services (Yahoo Answers), Google Wave, Search Engines and various vertical social networks are making it possible for people to access and contribute content, as well as collaborating over the web. While there are advancements in Web 2.0 [1], cloud computing, and service oriented architectures which have techniques, services, tools, content accessibility and sharing in general to foster collaboration, there no effective support to foster sharing and collaboration on course materials [2] and artifacts. These include co-editing slides and notes, sharing tutorials and references, reusing complementary materials, comments and feedbacks from external students and colleagues.

This demonstration paper presents a Web 2.0 social productivity platform, called Crowd Learning and Teaching (CrowdL\&T), which supports effective collaboration among teachers and students. The vision of CrowdL\&T is to promote a comprehensive, end-to-end framework and Web 2.0 social productivity platform [3] that unlock teaching and learning knowledge. This can be done by empowering teachers and students with robust techniques, efficient services, easy to use tools that allow them to share course artifacts and to collaborate on building a free knowledge base about teaching and learning based on lecture notes, slides, tutorials, video presentations, quizzes, and experiences. In addition, the CrowdL\&T platform aims to develop services for evaluating contribution of knowledge from collaborators.

\section{Architecture}

The following diagram depicts the overall architecture of our system. It consists of services, open APIs and portals that allow live interaction between users such as teachers and students. An open service is called CoreDB. It contains several modules. The first module is used as the basis for artifacts browsing and editing. The second module is used to enable traceability where changes to artifacts are traced. The third module is 


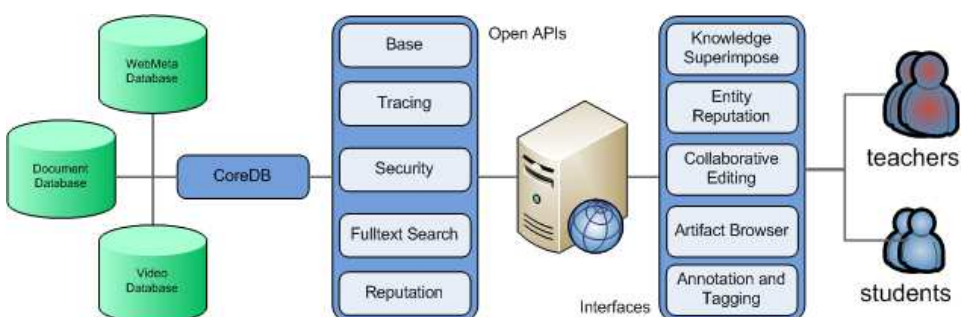

Fig. 1. Overall Architecture.

used for security where access control level (ACL) policy are created to manage access to entities representing artifacts and relationships. The forth module provides fulltext search capability over artifact database. Collaborative editing is a service provided by our system. It is a browser based tool that allows both private and live-collaborate editing of artifacts such as lecture notes and slides. Its functionalities include content importation from and exportation to well-known document formats (PDF and PowerPoint). Another feature that makes our system unique is tagging [4] artifacts with other various form of artifacts includes text books, research papers, video and audio lectures.

Another service provided by the system is knowledge improvement. This service is achieved by using the combination of the annotation tool and the creation of semantic relation technique that improve the second order knowledge. Another service is the artifact reputation. This service allows the system makes use of information from the sharing artifacts such as annotations, artifacts and artifact relations that compute the reputation of individual artifacts such as persons, files and folders. This improves search accuracy and provides incentives to users to collaborate.

\section{Demo Routine}

First, we show how teachers create, upload and organise courses. From a course workspace, teachers create course artifacts, namely lecture notes and slides using the notes collaborative editor featuring real-time. Users tag slides using terms automatically extracted from PDF, text books and online video lectures. Second, we show the organisation of course artifacts and the continuous superimposition of additional information through links to other artifacts forming dynamic folders. In doing this, the course knowledge base is incrementally enriched and provide valuable learning experiences to students and colleagues. Third, we demonstrate how feedback and ranking are used to improve artifacts quality and reputation. Demo Link: http://www . youtube.com/watch?v=yqBhQRFfinE

\section{References}

1. Ullrich, C., Borau, K., Luo, H., Tan, X., Shen, L., and Shen, R. 2008. Why web 2.0 is good for learning and for research: principles and prototypes. In Proc. WWW'08, pages 705-714.

2. Bercovitz, B., Kaliszan, F., Koutrika, G., Liou, H., Mohammadi Zadeh, Z., and Garcia-Molina, H. 2009. CourseRank: a social system for course planning. In Proc.SIGMOD'09, pages 1107-1110.

3. Huberman, B. A., Romero, D. M., and Wu, F. 2009. Crowdsourcing, attention and productivity. J. Inf. Sci. 35, 6 (Dec. 2009), 758-765.

4. Golder, S. A. and Huberman, B. A. 2006. Usage patterns of collaborative tagging systems. J. Inf. Sci. 32, 2 (Apr. 2006), 198-208. 